\title{
O transporte de carbono orgânico dissolvido e nutrientes nitrogenados no canal principal do Rio Amazonas, no Estreito de Óbidos
}

O presente trabalho foi realizado no Rio Amazonas, no Estreito de Óbidos, de janeiro de 2012 a dezembro de 2016. As coletas foram realizadas mensalmente com o objetivo de investigar as variações sazonais e interanuais do transporte de carbono orgânico dissolvido (COD), do nitrogênio total dissolvido (NDT), do nitrogênio orgânico dissolvido (DON), do nitrato (NO3) e do amônio (NH4) no rio. Observou-se que as fases hidrográficas têm uma influência importante sobre o transporte dos constituintes dissolvidos na água. Na fase de cheia, o carbono orgânico dissolvido (COD), o nitrogênio total dissolvido (NDT) e o nitrogênio orgânico dissolvido (DON) aumentaram significativamente seu transporte (sendo em média respectivamente $0.096,0.006$ e $0.004 \mathrm{Tg}$.dia-1), em comparação com a fase de seca (0.036, 0.003 e 0.001 Tg.dia-1, respectivamente). Apenas o nitrato (NO3) não teve seu transporte significativamente alterado ao longo das fases da hidrógrafa (0.003 Tg.dia-1). O transporte média de NH4 foi de $0.0003 \pm 0.0001 \mathrm{Tg}$.dia-1 e as diferenças no transporte foram serem negligenciadas. Os resultados encontrados no presente trabalho aproximam-se dos valores encontrados na literatura para o Rio Amazonas. Estes resultados demonstram que mudanças de cobertura e uso do solo ainda não podem ser observadas em larga escala na no Estreito de Óbidos. Somente um monitoramento contínuo e ininterrupto é capaz de fazer uma avaliação robusta do efeito de eventos climáticos extremos, possíveis mudanças futuras no clima, assim como mudanças no uso e cobertura do solo. Frente a crescentes mudanças na Bacia Amazônica, estudos com frequência temporal e ampla distribuição espacial fazem-se necessários.

Palavras-chave: Rio Amazonas; Carbono Orgânico Dissolvido; Nitrogênio; Amônio; Nitrato; Fluxo.

\section{The transport of dissolved organic carbon and nitrogen nutrients in the main channel of the Amazon River, in the Óbidos Strait}

\begin{abstract}
The present work had done in Amazon River mainstream, at Óbidos Narrow, from January 2012 to December 2016. The samples were carried out monthly with the objective of investigating the seasonal and interannual variations in load of dissolved carbon and nitrogened nutrients in the river. It was observed that the hydrograph phases have an important influence over transport of dissolved constituents in the water of river. At high water phase, dissolved organic carbon (DOC), total dissolved nitrogen (TDN) and dissolved organic nitrogen (DON) increased their transport (being on average $0.096,0.006$ e $0.004 \mathrm{Tg}$.day-1 respectively) when compared with low water phase $(0.036,0.003$ e $0.001 \mathrm{Tg}$.day-1, respectively). Just nitrate (NO3) did not change through the phases of hydrograph (0.003 Tg.dia1). The load of NH4 was on average $0.0003 \mathrm{Tg}$.dia-1 and differences in load were neglected. The results found in present work are close of those one found in literature for Amazon River mainstream. The results show that cover and land use changes cannot be observed in large scale yet. Only continuous and uninterrupted monitoring is capable of making a robust assessment of the effect of extreme climate events, as well a possible future changes in climate and cover and use land. Facing increasing changes in the Amazon Basin, studies with a temporal frequency and a wide spatial distribution are necessary.
\end{abstract}

Keywords: Amazon River; Dissolved Organic Carbon; Nitrogen; Ammonium; Nitrate; Load.

Topic: Hidrogeografia

Reviewed anonymously in the process of blind peer.
Received: 10/08/2018 Approved: 24/09/2018
Irene Cibelle Gonçalves Sampaio Universidade Federal do Oeste do Pará, Brasil http://lattes.cnpq.br/1181052395103997 irenecibelle@hotmail.com

Jose Mauro Sousa Moura (iD) Universidade Federal do Oeste do Pará, Brasil http://lattes.cnpq.br/1181052395103997 id.org/0000-0003-4962-8870 imaurosm@gmail.com

Robert Max Holmes (iD) Woods Hole Research Center, Estados Unidos http://orcid.org/0000-0002-6413-9154 rmholmes@whrc.org

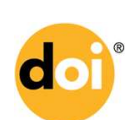

DOI: 10.6008/CBPC2179-6858.2018.007.0029
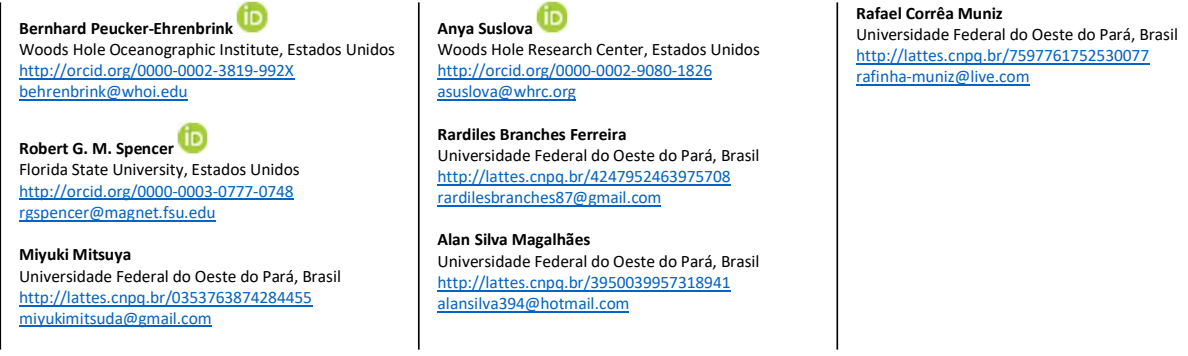

Referencing this:

SAMPAIO, I. C. G.; MOURA, J. M. S.; HOLMES, R. M.; PEUCKEREHRENBRINK, B.; SPENCER, R. G. M.; MITSUYA, M.; SUSLOVA, A.; FERREIRA, R. B.; MAGALHÃES, A. S.; MUNIZ, R. C.. O transporte de carbono orgânico dissolvido e nutrientes nitrogenados no canal principal do Rio Amazonas, no Estreito de Óbidos. Revista Ibero Americana de Ciências Ambientais, v.9, n.7, p.308-319, 2018. DOI: http://doi.org/10.6008/CBPC2179-6858.2018.007.0029 


\section{INTRODUÇÃO}

O Rio Amazonas é o maior rio do mundo em comprimento $\left(7 \times 10^{3} \mathrm{~km}\right)$, área de drenagem $(7.05 \mathrm{x}$ $10^{6} \mathrm{~km}^{2}$ ) e vazão (220800 $\mathrm{m}^{3} . \mathrm{s}^{-1}$ ) (ERTEL et al., 1986; MILLIMAN et al., 1983; RICHEY et al., 1986; SIOLI, 1984). Sozinho, é responsável por 15-20\% da água doce que chega aos oceanos (BARTHEN et al., 2004; FILIZOLA et al., 2009; MARTINELLI et al., 1989). Segundo Richey et al. (2002), os rios tropicais têm um maior impacto sobre o balanço global do carbono do que rios de regiões temperadas. A importância dos rios tropicais não é apenas por que são mais caudalosos, mas também porque possuem alta temperatura e baixa alcalinidade. Estes fatores potencializam as taxas de decomposição da matéria orgânica e a emissão de gases dissolvidos na água, como o $\mathrm{CO}_{2}$, para a atmosfera (ALKINS et al., 2001).

Por causa dessas características, Richey et al. (2002) acreditam que estudos sobre a dinâmica de carbono no Rio Amazonas devem ser a chave para o entendimento de algumas incongruências no balanço global de carbono. Isto porque, embora existam evidências de que a Amazônia seria um sumidouro de carbono, não se sabe onde este carbono estaria sendo 'estocado' (NOBRE et al., 2002).

Como os rios estão conectados ao ecossistema terrestre, mudanças na área de drenagem são um desafio para estudo da dinâmica do carbono no rio. Mudanças no uso e cobertura do solo podem alterar a dinâmica do carbono no rio por muitas razões. Pela alteração da quantidade e qualidade do carbono transportado do sistema terrestre para os rios, mudanças no clima, alterações físicas e químicas na água etc. Algumas dessas transformações já estão em curso e outras são previstas para breve, conforme estudos de Nobre et al. (2016) e McDowell et al. (2018).

De acordo com Nobre et al. (2016), a área desmatada na Amazônia já representa 20\% da área originalmente de floresta e nos últimos 60 anos a região amazônica já ficou $1^{\circ} \mathrm{C}$ mais quente. Ao longo de meio século a fronteira agrícola tem crescido sobre as áreas de floresta na Amazônia, resultando em mudanças significativas e generalizadas no uso e cobertura do solo. Estas mudanças têm aumentado o risco da conversão irreversível de uma vegetação de floresta tropical para uma vegetação de savana ('savanização'). Mudanças de floresta tropical para vegetação de savana, com menor biomassa, deve ter impacto no ciclo do carbono no ecossistema terrestre e aquático.

O desmatamento também possui impacto importante sobre as áreas adjacentes as áreas desmatadas. Aparentemente, em todas as florestas tropicais úmidas está ocorrendo um aumento nas taxas de mortalidade de árvores, associado à elevação da temperatura, déficit de pressão de vapor, seca, abundância de lianas, eventos de vento, fogo e possivelmente a um aumento do $\mathrm{CO}_{2}$ na atmosfera (MCDOWELL et al., 2018).

Tanto a 'savanização' quanto o aumento da mortalidade das árvores têm um potencial impacto na quantidade e na qualidade da matéria orgânica transportada dos ecossistemas terrestres para os rios. Assim como sobre o balanço de carbono. Embora existam estudos importantes sobre a dinâmica do carbono no Rio Amazonas (DEVOL et al., 1987; RICHEY et al., 1990, 2002; MARTINELLI et al., 1991; QUAY et al., 1992; DEVOL 
et al., 2001; MCCLAIN et al., 2001, 2001; MAYORGA et al., 2005; WARD et al., 2015), todos eles são baseados em amostra coletadas em cruzeiros (temporalmente pontual).

As amostragens baseadas em cruzeiros (com frequências baixas; anuais ou semestrais) são importantes para entender a espacialidade das variações da dinâmica do carbono ao longo do Rio Amazonas. Esse tipo de amostragem, no entanto, não é robusto o suficiente para entender a variação da quantidade de carbono transportado ao longo da hidrógrafa e variações interanuais. Estas amostragens, com baixa frequência temporal, também não são adequadas para identificar alterações ligadas às mudanças da cobertura e uso do solo ou de mudanças climáticas futuras. Apenas um conjunto de dados longo e temporalmente frequente pode identificar um padrão do transporte de carbono orgânico e de compostos nitrogenados dissolvidos no rio, e suas possíveis alterações. Por isso, o presente estudo foi conduzido mensalmente em cinco anos ininterruptos. Esta é uma das maiores séries temporais de COD e de compostos nitrogenados dissolvidos no canal principal do Rio Amazonas, e a única com amostragens mensais. A análise desse dados tem por objetivo compreender a dinâmica natural de transporte de carbono orgânico dissolvido e compostos nitrogenados no canal principal do Rio Amazonas, na região do estreito de Óbidos.

\section{METODOLOGIA}

\section{Amostragem e Análise Laboratorial}

As coletas foram realizadas no Rio Amazonas, na região do estreito de Óbidos, a montante da cidade de Óbidos. O presente trabalho analisou amostras mensais do período de janeiro de 2012 a dezembro de 2016. As análises de carbono orgânico dissolvido (COD), nitrogênio orgânico dissolvido (NOD), nitrogênio total dissolvido (NTD), Nitrato $\left(\mathrm{NO}_{3}\right)$ e amônio $\left(\mathrm{NH}_{4}\right)$ dissolvidos na água do rio foram baseadas em amostras composta. Ou seja, mensalmente, a água foi coletada em três diferentes pontos do rio e reunida em uma única amostra. No centro do rio foi coletado dois litros de água. Em dois pontos equidistantes, em relação às margens e ao ponto central, foram coletados um litro em cada ponto. Cada amostra foi composta, portanto, por 4 litros de água superficial.

Após a coleta, a amostra foi armazenada em garrafa de HDPE (High-density polyethylene - polietileno de alta densidade) e colocada em isopor. No laboratório as amostras foram filtradas (filtro <0,45 $\mu \mathrm{m}$ ), armazenadas em garrafas de HDPE (60 ml) e congeladas até a análise química.

O carbono orgânico dissolvido (COD) foi determinado através de combustão de alta temperatura utilizando um analisador de carbono orgânico Shimadzu TOC-V. A precisão geral das repetições independentes foi < 5\% (MANN et al., 2012). O nitrogênio total dissolvido (NDT), nitrogênio orgânico dissolvido (DON), nitrato + dióxido de nitrogênio $\left(\mathrm{NO}_{3}+\mathrm{NO}_{2}\right)$ e amônio $\left(\mathrm{NH}_{4}\right)$ foram determinados pelo método colorimétrico, utilizando um analisador Astoria $2^{\circledR}$.

A temperatura da água foi medida diretamente em campo, aproximadamente $50 \mathrm{~cm} \mathrm{de}$ profundidade, por um sensor YSI Profissional ${ }^{\circledR}$. Para o cálculo da carga transportada pelo rio foram utilizados a concentração dos constituintes estudados e dados de vazão do rio. A concentração dos constituintes foi 
obtida elas análises descritas acima, já os dados de vazão foram obtidos na página do projeto Hybam obtido da estação fluviométrica da Agência Nacional de Água (ANA), localizada em frente à cidade de Óbidos (Estação código: 17050001).

A estimativa do transporte dos constituintes dissolvidos em água (COD, NDT, NOD, $\mathrm{NO}_{3}$ e $\mathrm{NH}_{4}$ ) foi realizada com o uso do programa LOADRunner. Este programa foi desenvolvimento pela Universidade de Yale/USA (Yale School of Forestry and Environmental Science), com auxílio do Woods Hole Research Center (WHRC).

\section{Análise Estatística}

As variáveis foram primeiramente testadas quanto a sua distribuição, através do teste de ShapiroWilk. A normalidade foi rejeitada quando o valor de $p$ foi menor que 0.05 . Para comparar os períodos amostrados (fases hidrógrafa ou anos) foi utilizado o teste ANOVA-One Way ou Kruskal-Wallis. Apenas os dados de temperatura da água apresentaram distribuição normal. Neste caso, foi utilizado o teste ANOVAOne Way. Para os demais parâmetros, que não apresentaram distribuição normal, foi utilizado o teste não paramétrico de variância de Kruskal-Wallis, seguido pelo teste Dunn.

\section{RESULTADOS}

Com base na série histórica (1969-2016), a quantidade total de água (somatória) que passa anualmente em frente à cidade de Óbidos é em média $5512 \pm 476 \mathrm{Km}^{3}$.

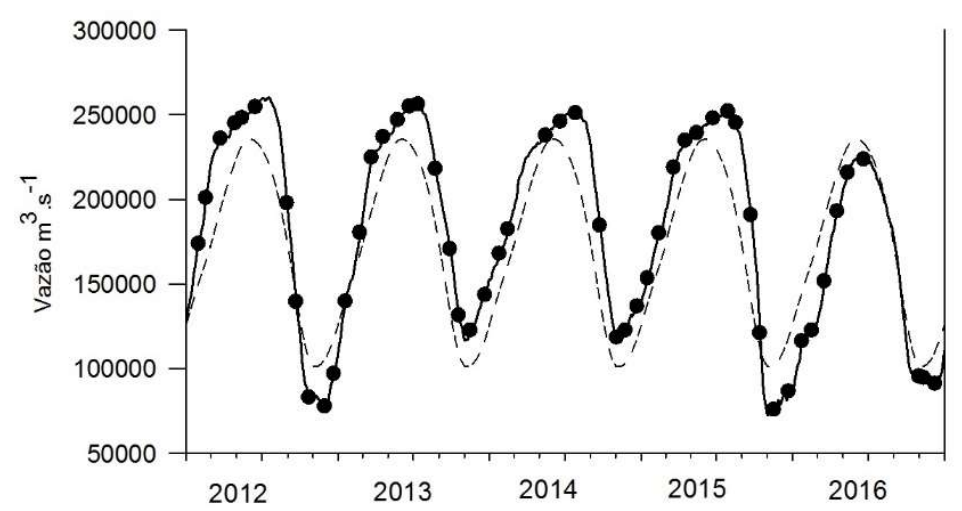

Figura 1: Vazão de água do Rio Amazonas, na frente da cidade de Óbidos. O gráfico ' $A$ ' demonstra a série história da vazão (1969 a 2016). A linha pontilhada vermelha representa a vazão em anos de EL Nino. O Gráfico 'B' a vazão nos anos do presente estudo. Os pontos representam os valores da vazão nos dias de coleta e a linha pontilhada representa a média da série histórica.

As fases da hidrógrafa foram determinadas pela média da série histórica para cada mês. Analisando essas médias, foi identificada uma fase de seca (com meses cujos valores da vazão média foram menores que o valor limite do primeiro quartil), fase de cheia (com valores superiores ao valor limite do terceiro quartil), fase de enchente e fase de vazante, período com valores de vazão intermediários. Com base nesse método, foi possível identificar que o padrão da fase cheia ocorre de maio a julho e a fase de seca de outubro a dezembro. Existe uma longa fase de enchente com quatro meses (janeiro a abril) e uma rápida fase de 
vazante que ocorre em dois meses (agosto e setembro). A vazão ao longo dos anos deste estudo pode ser observada na Figura 1. Também não foi encontrada diferença na temperatura da água, quando comparamos os anos do presente estudo (ANOVA $p>0,05$ ). A estimativa do transporte dos constituintes é apresentada na Tabela 1 por fase da hidrógrafa em cada um dos anos do presente estudo.

Tabela 1: Transporte total de COD, NOD, NDT, $\mathrm{NO}_{3}$ e $\mathrm{NH}_{4}(\mathrm{Tg})$ e vazão ' $\mathrm{Q}$ ' $\left(\mathrm{Km}^{3}\right)$ em cada fase da hidrógrafa e o total transportado para cada ano do presente estudo (2012-2016).

\begin{tabular}{|c|c|c|c|c|c|c|}
\hline Constituintes & 2012 & 2013 & 2014 & 2015 & 2016 & Média \\
\hline \multicolumn{7}{|c|}{ Jan-Abr/Enchente } \\
\hline$Q$ & 2136 & 1940 & 2036 & 2007 & 1476 & 1919 \\
\hline COD & 10.7 & 8.2 & 7.9 & 8.3 & 7.2 & 8.5 \\
\hline NOD & 0.6 & 0.4 & 0.3 & 0.3 & 0.3 & 0.4 \\
\hline NDT & 0.9 & 0.8 & 0.8 & 0.7 & 0.6 & 0.7 \\
\hline $\mathrm{NO}_{3}$ & 0.3 & 0.5 & 0.02 & 0.4 & 0.4 & 0.32 \\
\hline $\mathrm{NH}_{4}$ & 0.01 & 0.02 & 0.5 & 0.02 & 0.04 & 0.1 \\
\hline \multicolumn{7}{|l|}{ Mai-Jul/Cheia } \\
\hline $\mathrm{Q}$ & 2015 & 1984 & 1943 & 1950 & 1738 & 1926 \\
\hline COD & 9.8 & 8.3 & 7.9 & 8.7 & 9.6 & 8.9 \\
\hline NOD & 0.5 & 0.4 & 0.3 & 0.3 & 0.3 & 0.4 \\
\hline NDT & 0.7 & 0.6 & 0.6 & 0.5 & 0.5 & 0.6 \\
\hline $\mathrm{NO}_{3}$ & 0.2 & 0.3 & 0.3 & 0.2 & 0.2 & 0.3 \\
\hline $\mathrm{NH}_{4}$ & 0.02 & 0.02 & 0.03 & 0.03 & 0.04 & 0.03 \\
\hline \multicolumn{7}{|c|}{ Ago-Set/Vazante } \\
\hline$Q$ & 1026 & 1101 & 1180 & 1185 & 906 & 1080 \\
\hline COD & 4.3 & 4.1 & 4.4 & 5.0 & 4.8 & 4.50 \\
\hline NOD & 0.2 & 0.2 & 0.2 & 0.2 & 0.2 & 0.2 \\
\hline NDT & 0.3 & 0.3 & 0.3 & 0.3 & 0.3 & 0.3 \\
\hline $\mathrm{NO}_{3}$ & 0.2 & 0.2 & 0.1 & 0.1 & 0.02 & 0.1 \\
\hline $\mathrm{NH}_{4}$ & 0.01 & 0.01 & 0.01 & 0.01 & 0.1 & 0.03 \\
\hline \multicolumn{7}{|l|}{ Out-Dez/Seca } \\
\hline$Q$ & 452 & 692 & 667 & 426 & 502 & 548 \\
\hline COD & 1.6 & 2.3 & 2.3 & 1.7 & 2.6 & 2.1 \\
\hline NOD & 0.1 & 0.1 & 0.1 & 0.03 & 0.1 & 0.1 \\
\hline NDT & 0.2 & 0.2 & 0.2 & 0.1 & 0.2 & 0.2 \\
\hline $\mathrm{NO}_{3}$ & 0.1 & 0.2 & 0.2 & 0.1 & 0.1 & 0.1 \\
\hline $\mathrm{NH}_{4}$ & 0.004 & 0.01 & 0.01 & 0.01 & 0.01 & 0.01 \\
\hline \multicolumn{7}{|l|}{ ANUAL } \\
\hline $\mathrm{Q}$ & 5629 & 5717 & 5826 & 5568 & 4622 & 5472 \\
\hline COD & 26.5 & 22.9 & 22.5 & 23.7 & 24.3 & 23.9 \\
\hline NOD & 1.4 & 1.1 & 0.9 & 0.8 & 0.8 & 1.0 \\
\hline NDT & 2.0 & 1.9 & 1.8 & 1.6 & 1.5 & 1.8 \\
\hline $\mathrm{NO}_{3}$ & 0.8 & 1.1 & 0.6 & 0.8 & 0.6 & 0.8 \\
\hline $\mathrm{NH}_{4}$ & 0.04 & 0.1 & 0.6 & 0.1 & 0.2 & 0.2 \\
\hline
\end{tabular}

Para todos os constituintes estudados, não houve diferença na carga anual transportada entre os anos do presente estudo (2012-2016) (Kruskal-Wallis p>0.05)(Tabela 1). Observou-se que cerca de $70 \%$ do COD é transportado na enchente-cheia (janeiro-julho). Em média, a quantidade de COD transportada na fase de enchente é de $0.07 \pm 0.02 \mathrm{Tg}$. dia ${ }^{-1} \mathrm{e}$ na fase de cheia $0.096 \pm 0.009 \mathrm{Tg}$. dia ${ }^{-1}$. Este transporte na enchentecheia é significativamente maior que o transporte na fase de seca, que corresponde a $0.036 \pm 0.008 \mathrm{Tg}$. dia ${ }^{-1}$ (Kruskal-Wallis; Dunn $\mathrm{p}<0.05$ ). No período deste estudo, os valores máximos de transporte de COD foi 0.11 Tg. dia ${ }^{-1}$ e mínimo de 0.02 Tg. dia ${ }^{-1}$.

A quantidade de NOD transportado na cheia $\left(0.004 \pm 0.001 \mathrm{Tg}\right.$. dia $\left.{ }^{-1}\right)$ é maior do que em na seca $\left(0.001 \pm 0.0004 \mathrm{Tg}\right.$ dia $\left.^{-1}\right)($ Kruskal-Wallis; Dunn $p<0.05)$. Outras fases não foram diferentes entre si (Dunn $p>$ 
0,05); Sendo, em média, o transporte de NOD na enchente e vazante respectivamente: $0.003 \pm 0.001 \mathrm{Tg}$. dia ${ }^{1}$ e $0.003 \pm 0.001 \mathrm{Tg}$. dia ${ }^{-1}$. Os valores máximos e mínimos de transporte de NOD foram respectivamente 0.006 e 0.0004 Tg. dia ${ }^{-1}$.
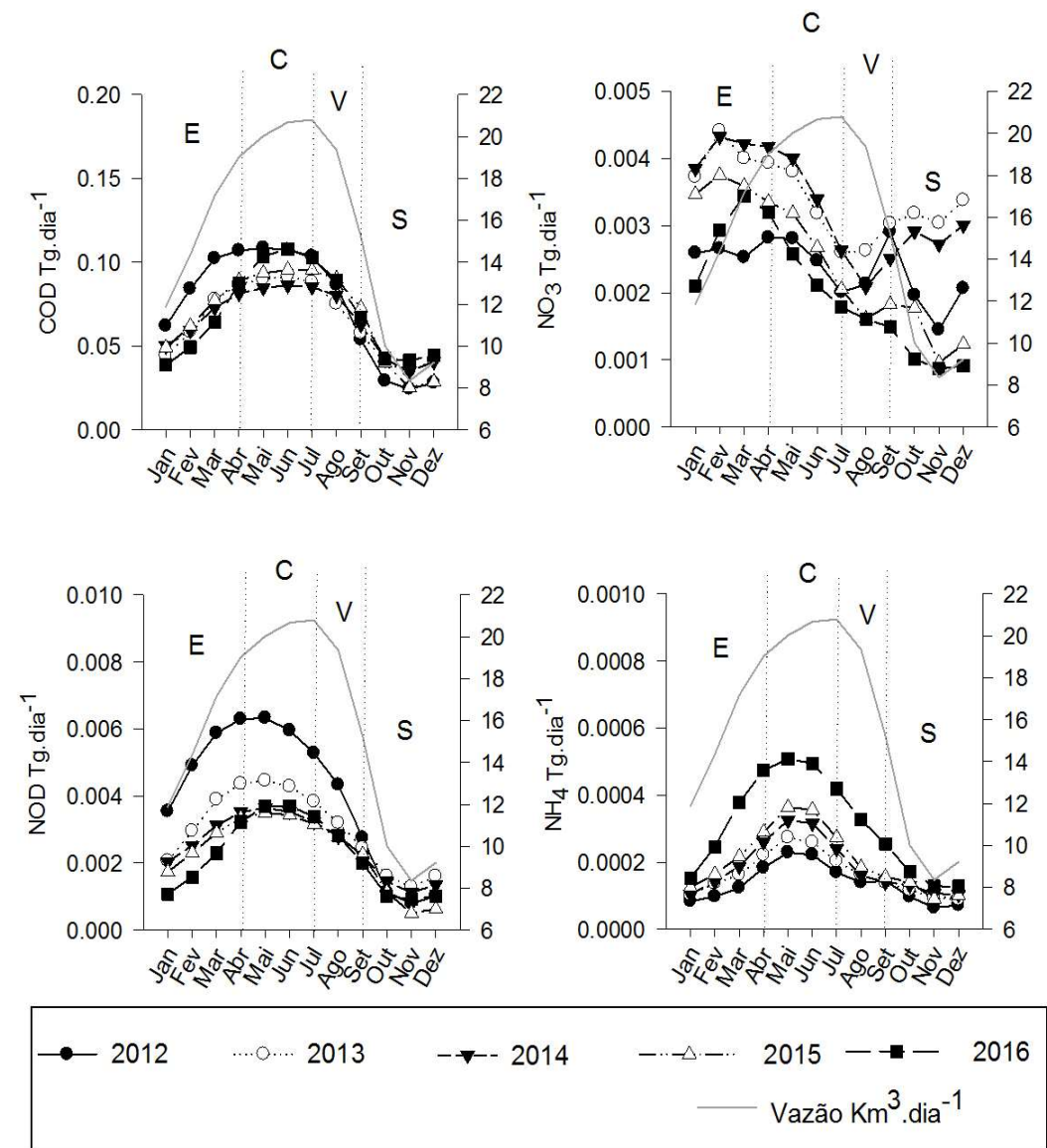

Figura 2: Variação mensal no transporte DOC, DON, NDT, $\mathrm{NH}_{4}$ e $\mathrm{NO}_{3}$ no Estreito de Óbidos (2012-2016). A média da vazão nos anos do presente estudo é mostrada com eixos ' $Y$ ' à direita de cada gráfico $\left(\mathrm{Km}^{3}\right.$. dia' $\left.{ }^{-1}\right)$ e representada pela linha cinza. Linhas pontilhadas verticais representam a separação entre a fase hidrográfica. As letras E, C, V e $S$ representam, respectivamente, as fases de enchente, cheia, vazante e seca.

Não houve diferença no transporte médio de NDT na enchente $\left(0.006 \pm 0.001 \mathrm{Tg}\right.$. dia $\left.{ }^{-1}\right)$ e cheia $(0.006$ $\left.\pm 0.001 \mathrm{Tg}^{\mathrm{dia}}{ }^{-1}\right)$, mas ambos são diferentes do fluxo de NDT na seca $\left(0.003 \pm 0.001 \mathrm{Tg}\right.$. dia $\left.{ }^{-1}\right)$ (Kruskal-Wallis; Dunn $p<0,05)$.Sendo 0.008 e $0.002 \mathrm{Tg}$. dia ${ }^{-1}$, repectivamente, os valores máximo e mínimo do transporte de NDT. O transporte de $\mathrm{NO}_{3}$ não diferiu ao longo da fase hidrográfica, sendo $0.003 \pm 0.001,0.003 \pm 0.001,0.002$ \pm 0.001 e $0.002 \pm 0.001 \mathrm{Tg}$. dia ${ }^{-1}$ (Kruskal-Wallis $p>0.05$ ). Os valores máximo e mínimo de transporte de NO3 foram respectivamente 0.004 e $0.0008 \mathrm{Tg}$. dia-1. A quantidade de $\mathrm{NH}_{4}$ transportada pelo rio é pequena e suas diferenças ao longo da hidrógrafa podem ser negligenciadas, sendo em média $0.0003 \pm 0.0001 \mathrm{GgC}$.dia-1 , com valores máximo e mínimo de 0.0005 e $0.0001 \mathrm{Tg}$. dia ${ }^{-1}$, respectivamente (Figura 2).

A forma mais abundante de $\mathrm{N}$ no rio foi NOD, representando em cerca de $50 \%$ do NDT. A razão COD:NOD foi maior na fase de seca $(46,75 \pm 14,29)$ do que de enchente $(23,53 \pm 6,61)$ (Krushkal; Dunn $p$ $<0,05)$. Cheia $(26,98 \pm 4.17)$ e vazante $(26,74 \pm 4,29)$ não diferiram de outras fases da hidrógrafa (Krushkal; Dunn $p>0,05)$. Relação NOD:NDT diminuiu na fase seca $(0,31 \pm 0,20)$, quando comparado com a enchente 
$(0.52 \pm 0.15)$ e cheia $(0.62 \pm 0.06)$ (Kruskal-Wallis; Dunn $p<0,05)$. A fase de vazante $(0.54 \pm 0.06)$ não diferiu de outras fases (Krushkal; Dunn $p>0,05)$.

\section{DISCUSSÃO}

Os dados do presente estudo não mostraram alterações na vazão, fluxo de $\mathrm{COD}, \mathrm{NOD}, \mathrm{NDT}, \mathrm{NO}_{3}$ ou $\mathrm{NH}_{4}$ ou temperatura da água ao longo dos anos (2012-2016). Estudos realizados em pequenas bacias encontraram um impacto da mudança da cobertura e uso da terra sobre a dinâmica de carbono e nitrogênio dissolvido, vazão e temperatura na água do rio (BARROSO et al., 2017).

Com a conversão de florestas em áreas de atividade agropecuária, é esperado que o transporte de matéria orgânica da área de drenagem para o rio seja reduzido. Isto porque a biomassa vegetal acima do solo, que compõem a vegetação de atividades agropecuárias, tem uma biomassa menor que a da floresta (SOLAR et al., 2016).

A principal forma de aporte de $\mathrm{N}$ na Bacia Amazônica é a fixação biológica de $\mathrm{N}$ em ecossistemas naturais (FILOSO et al., 2006; MARTINELLI et al., 2010). A quantidade de compostos nitrogenados inorgânicos $\left(\mathrm{NO}_{3}\right.$ e $\left.\mathrm{NH}_{4}\right)$ transportado pelo rio tende a ser reduzidos após a conversão de floresta para área com atividades agropecuárias (DIAS-FILHO et al., 2001; NEILL et al., 2001; FIGUEIREDO et al., 2010). A redução do transporte de nitrogênio, em rios cuja bacia de drenagem é ocupada por atividades agropecuárias, pode ser explicada pela redução do nitrogênio no solo (GROPPO et al., 2015) (a menos que haja adubação química).

Outra explicação para a redução do transporte de compostos nitrogenados, nos rios com área de drenagem sob atividade agropecuária, pode ser o menor transporte de matéria orgânica para os rios. Este segundo fator pode afetar tanto o transporte de carbono quanto de nitrogênio no rio. Isto porque, durante a respiração do carbono (matéria orgânica) existe a produção tanto de $\mathrm{CO}_{2}$ como de $\mathrm{NO}_{3}$ (DEVOL et al., 2001). Portanto, a redução de biomassa vegetal pode resultar na redução do transporte de matéria orgânica para o rio, e consequente redução da $\mathrm{COD}, \mathrm{CO}_{2}$ dissolvido e $\mathrm{NO}_{3}$ transportados pelo rio.

Richey et al. (1996) afirmam que a remineralizarão do nitrogênio é rapidamente nitrificado para nitrato. Segundo estes autores, esta seria a explicação do fato do fluxo de $\mathrm{NO}_{3}$ no estreito de Óbidos ser aproximadamente $30 \%$ maior que ao acumulo do aporte a montante. Apesar de mudanças nos compostos nitrogenados (NOD, $\mathrm{NO}_{3}, \mathrm{NH}_{4}$ ) terem sido encontradas em rios de pequeno porte (DIAS-FILHO et al. 2001; FILOSO et al., 2006; MARTINELLI et al., 2010) , estas mudanças ainda não são observadas no canal principal do rio Amazonas no Estreito de Óbidos; larga escala. Isso pode ocorrer pelo fato de que rios menores, geralmente, estão mais conectados ao ambiente terrestre e refletem mais rapidamente mudanças que ocorrem em sua bacia de drenagem.

As áreas mais densamente desmatadas da Amazônia estão localizadas em uma região chamada 'Arco do Desmatamento'. Nesta região de fronteira agrícola, os impactos da mudança da cobertura e do uso da terra já podem ser vistos nos parâmetros físico-químicos do rio da água. No entanto, a região da bacia amazônica, que drena as águas a montante de Óbidos, ainda está relativamente bem preservada. 
Os valores de $\mathrm{COD}$ e de compostos nitrogenado (NOD, $\mathrm{NO}_{3}$ e $\mathrm{NH}_{4}$ ) do presente estudo estão em escala de grandeza semelhante ao de estudos anteriores no Estreito de Óbidos (Tabela 2) (RICHEY et al., 1990; RICHEY et al., 1991; MARTINELLI et al., 1991; HEDGES et al., 2000; MOREIRA-TURCQ et al., 2003). No entanto, não se recomenda o uso de amostras temporalmente pontuais no estudo de dinâmica de carbono e nitrogênio no Rio Amazonas. Diante de mudanças iminentes, como a 'savanização' de áreas na Amazônia e mudanças climáticas (LOVEJOY et al., 2018), apenas o monitoramento mensal será capaz de identificar os primeiros impactos de mudanças em larga escala.

É possível que os primeiros impactos na dinâmica do carbono e do nitrogênio no Estreito de Óbidos sejam observados devido eventos de anomalias climáticas e não unicamente devido à mudança na cobertura e uso do solo. Isso porque as anomalias climáticas podem atingir grandes áreas em pouco tempo. Além disso, estes são fenômenos repentinos têm força e alcance imprevisíveis. O fato de a Bacia Amazônica a montante de Óbidos estar relativamente bem preservada oferece uma excelente oportunidade para caracterizar padrões naturais e identificar efetivamente possíveis mudanças futuras, seja devido a mudanças na cobertura ou uso da terra ou devido às mudanças climáticas.

Durante o presente estudo ocorreram fortes anomalias climáticas na Amazônia relacionados a eventos de La Niña, El Niño e aquecimento da temperatura da superfície do mar (STT) no Atlântico tropical ao sul do Equador (MARENGO et al., 2008; 2012; 2013; ESPINOZA et al., 2013; SATYAMURTY et al., 2013; SATYAMURTY et al., 2013; MARENGO et al., 2016).

Estes eventos provocaram fortes enchentes na Amazônia em 2012 (MARENGO et al., 2016; LOVEJOY et al., 2018). Em 2014 e 2015 também foram observadas inundações, na região sudoeste da Amazônia (LOVEJOY et al., 2018), devido um aumento em 100\% das chuvas de verão, que normalmente precipitam sobre a região, e atingiram níveis recordes na subida do nível da água dos rios Madeira e Rio Branco (MARENGO et al., 2016).

Um forte El Niño foi responsável por fazer de 2016 o ano mais quente do século passado, na Região Amazônica (JIMÉNEZ-MUÑOZ et al., 2016). No entanto, comparando a temperatura da água no Estreito de Óbidos em 2016 com os demais anos deste estudo, é possível dizer que o fenômeno não foi capaz de alterar a temperatura da água, cuja média foi de $29,3 \pm 0,9{ }^{\circ} \mathrm{C}$ (ANOVA $\mathrm{p}>0,05$ ). Este fator é importante, pois diferenças de temperatura pode influenciar na decomposição da matéria orgânica (ALKINS et al., 2001).

Os fenômenos de El Niño terem sido relacionados redução da precipitação, redução da vazão do rio e fonte de carbono em floresta não perturbadas da Amazônia (o que poderia afetar o transporte de carbono para os rios) (RICHEY et al., 1986; NOBRE et al., 2002; LIU et al., 2017) e os fenômenos de El Niña terem sido relacionados a enchentes e sumidouro de carbono na Floresta Amazônica (NOBRE et al., 2002; LOVEJOY et al., 2018). No entanto, nenhuma dessas anomalias climáticas (observadas durante este estudo 2012-2016) alteraram significativamente a vazão ou a quantidade de $\mathrm{COD}, \mathrm{NOD}, \mathrm{NDT} \mathrm{NO}_{3}$ e $\mathrm{NH}_{4}$ transportados no Estreito de Óbidos. 
Tabela 2: Comparação da concentração e fluxo de DOC do presente estudo com outros estudos em rios tropicais. Nas referências, * representa o presente estudo, 1 (COYNEL et al., 2005), 2 (DEVOL et al., 2001), 3 (HUANG et al., 2012), 4 (MARTINELLI et al., 2010), 5 (MOREIRA-TURCQ et al., 2003), 6 (RICHEY et al., 1990) e 7 (RICHEY et al., 1991). Considerando Devol et al. (2001) que afirmam que os valores de DIN são virtualmente o de NO3, para efeitos de comparação, os valores de transporte de DIN do estudo de Martinelli et al. (2010) e Lewis et al. (1999) foram considerados como de $\mathrm{NO}_{3}$.

\begin{tabular}{|c|c|c|c|c|c|c|}
\hline River & $\begin{array}{l}\text { Vazão } \\
\mathrm{m}^{3} \cdot \mathrm{s}^{-1} \\
\end{array}$ & $\begin{array}{l}\text { DOC } \\
\text { TgC.ano-1 }\end{array}$ & $\begin{array}{l}\text { DON } \\
\text { TgC.ano }{ }^{-1}\end{array}$ & $\begin{array}{l}\mathrm{NO}_{3} \\
\text { TgC.ano-1 }\end{array}$ & $\begin{array}{l}\mathrm{NH}_{4} \\
\text { TgC.ano-1 }\end{array}$ & Ref. \\
\hline Amazonas -Óbidos & 193052 & 27.35 & 1.47 & 0.87 & 0.05 & $* / 2012$ \\
\hline Amazonas -Óbidos & 196823 & 23.94 & 1.09 & 1.24 & 0.06 & $* / 2013$ \\
\hline Amazonas -Óbidos & 186608 & 23.77 & 0.93 & 1.21 & 0.07 & $* / 2014$ \\
\hline Amazonas -Óbidos & 154502 & 24.9 & 0.84 & 0.89 & 0.07 & $* / 2015$ \\
\hline Amazonas -Óbidos & 154502 & 25.6 & 0.82 & 0.73 & 0.11 & $* / 2016$ \\
\hline Amazonas -Óbidos & 157142 & & 0.73 & & & 4 \\
\hline Amazonas-Óbidos & & 19.1 & & & & 7 \\
\hline Amazonas-Óbidos & & 22 & & & & 2 \\
\hline Amazonas-Óbidos & & 22.1 & & & & 6 \\
\hline Amazonas-Óbidos & & 27.1 & & & & 5 \\
\hline Amazonas-Óbidos & & & 0.73 & $(0.85)$ & & 4 \\
\hline Amazonas & & 25 & & 0.81 & & 2 \\
\hline Solimões Manacapuru & 98625 & & 0.49 & & & 4 \\
\hline Iça & 7312 & & 0.04 & & & 4 \\
\hline Jutaí & 3425 & & 0.03 & & & 4 \\
\hline Juruá & 3737 & & 0.02 & & & 4 \\
\hline Japurá & 16000 & & 0.07 & & & 4 \\
\hline Purus & 11862 & & 0.08 & & & 4 \\
\hline Negro & 28937 & & 0.28 & & & 4 \\
\hline Madeira & 25878 & & 0.12 & & & 4 \\
\hline Congo (Zaire) & & 12.4 & & & & 1 \\
\hline Rios Tropicais - África & & 28 & & & & 3 \\
\hline Rios Tropicais /Américas & & 58.2 & & & & 3 \\
\hline Rios Tropicais/Ásia & & 45 & & & & 3 \\
\hline Rios Tropicais /Oceania & & 4.48 & & & & 3 \\
\hline Rios Tropicais/Global & & 136 & & & & 3 \\
\hline
\end{tabular}

Os dados o presente estudo demonstram uma forte ligação entre o transporte dos constituintes estudados e a hidrógrafa. Portanto, alterações na quantidade ou no deslocamento das fases da hidrógrafa, provocado por eventos climáticos extremos, devem ter efeito indireto sobre o transporte de carbono e nitrogênio pelo rio.

\section{CONCLUSÕES}

A dinâmica do carbono orgânico e nitrogênio dissolvido estão claramente ligados ao ciclo hidrológico. O transporte de carbono orgânico e nitrogênio dissolvido aumentou no período de enchente-cheia. Os valores de carga de carbono orgânico e nitrogênio dissolvidos, transportados no Estreito de Óbidos, foram semelhantes aos valores encontrados por trabalhos que foram realizados por cruzeiros.

Não foi observada qualquer mudança na dinâmica do $\mathrm{COD}, \mathrm{NOD}, \mathrm{NO}_{3}$ e $\mathrm{NH}_{4}$, em larga escala, que pudesse ser relacionada à mudanças de cobertura e uso da terra ao longo dos anos. Os fenômenos climáticos de 2012 (La Niña) e de 2015-2016 (El Niño) não interferiram na quantidade total de COD , NOD, TDN, $\mathrm{NO}_{3}$ e $\mathrm{NH}_{4}$ transportado pelo rio no ano. Não é possível afirmar, no entanto, que outros eventos extremos ou outros fenômenos da mudança climática não alterarão essa dinâmica. Apenas com uma amostragem longa e 
frequente, é possível fornecer uma base robusta para a análise da futura cobertura vegetal e uso da terra da bacia; e mudanças climáticas futuras.

AGRADECIMENTO: Ao Woods Hole Research Center (WHRC) pelo financiamento do projeto e auxílio científico, a Universidade Federal do Oeste do Pará (UFOPA) apoio científico e logístico, FADESPA pela concessão de bolsa de doutorado, CAPES pela bolsa de doutorado sanduíche (processo 88881.135432/201601) e CNPq pelas bolsas de iniciação científica.

\section{REFERÊNCIAS}

ALKINS, P.; JONES, L.. Princípios de Química. Porto Alegre: Bookman, 2001.

BARROSO, D. F. R.; FIGUEIREDO, R. O.; PIRES, C. S.; COSTA, F. F.. Impactos de diferentes usos da terra sobre os recursos hídricos em microbacias no Nordeste Paraense na Amazônia Oriental. In: Impactos do uso da terra em diferentes escalas. Belém: EMBRAPA Oriental, 2017. p.339-368.

BARTHEN, R. B.; CHARVET-ALMEIDA, P.; MONTAC, L. F. A.; LANNA, A. E.. Amazon Basin GIWA: Regional Assessment 40 b. Nairobi: University of Kalmar on behalf of United Nations Enviroment Programme, 2004.

COYNEL, A.; SEYLER, P.; ETCHEBER, H.; MEYBECK, M.; ORANGE, D.. Spatial and Seasonal Dynamics of Total Suspended Sediment and Organic Carbon Species in the Congo River. Global Biogeochemical Cycles, v.19, n.4, p.GB4019, 2005. DOI: http://doi.org/10.1029/2004GB002335

DEVOL, A. H.; HEDGES, J. I.. Organic matter and nutrients in the mainstem Amazon river. In: MCCLAIN, M. E.; VICTORIA, R.; RICHEY, J. E.. The Biogeochemistry of the Amazon Basin. New York: Oxford University Press, 2001.

DEVOL, A. H.; QUAY, P. D.; RICHEY, J. E.; MARTINELLI, L. A.. The Role of Gas Exchange in the Inorganic Carbon, Oxygen, and 222Rn Budgets of the Amazon River1. Limnology and Oceanography, v.32, n.1, p.235-248, 1987. DOI: http://doi.org/10.4319/lo.1987.32.1.0235

DIAS-FILHO, M. B.; DAVIDSON, E. A.; CARVALHO, C. J. R.. Linking Biogeochemical Cycles to Cattle Pasture Management and Sustainability in the Amazon Basin. In: The Biogeochemistry of the Amazon Basin. New York: Oxford University Press, 2001.

ERTEL, J. R.; HEDGES, J. I.; DEVOL, J.; RIBEIRO, M. N. G.. Dissolved humic substances of the Amazon River system. Limnology Oceanography, v.31, n.4, p.739-754, 1986. DOI: http://doi.org/10.4319/lo.1986.31.4.0739

ESPINOZA, J. C.; RONCHAIL, J.; FRAPPART, F.; LAVADO, W.; SANTINI, W.; GUYOT, J. L.. The Major Floods in the Amazonas River and Tributaries (Western Amazon Basin) during the 1970-2012 Period: A Focus on the 2012 Flood. Journal of Hydrometeorology, v. 14, n. 3, p. 1000-1008, jun. 2013. DOI: http://doi.org/10.1175/JHM-D-12-0100.1
FIGUEIREDO, R. O.; MARKEWITZ, D.; DAVIDSON, E. A.; SCHULER, A. E.; DOS S. WATRIN, O.; DE SOUZA SILVA, P.. Land-Use Effects on the Chemical Attributes of Low-Order Streams in the Eastern Amazon. Journal of Geophysical Research, v.115, n.G4, 2010. DOI: http://doi.org/10.1029/2009JG001200

FILIZOLA, N.; CÂNDIDO, L.; MANZ, A.; ESPINOZA, J. C.; RONCHAIL, J.; GUYOT, J. L.. Variabilidade hidrológica na Amazônia, uma perspectiva para a elaboração do balanço hídrico regional. In: Amazonía y agua: desarrollo sostenible en el siglo XXI. 2009. p.109-117.

FILOSO, S.; MARTINELLI, L. A.; HOWARTH, R. W.; BOYER, E. W.; DENTENER, F.. Human activities changing the nitrogen cycle in Brazil. In: Nitrogen Cycling in the Americas: Natural and Anthropogenic Influences and Controls. Springer, 2006. p.61-89.

GROPPO, J. D.; LINS, S. R. M.; CAMARGO, P. B.; ASSAD, E. D.; PINTO, H. S.; MARTINS, S. C.; SALGADO, P. R.; EVANGELISTA, B.; VASCONCELLOS, E.; SANO, E. E.; PAVÃO, E.; LUNA, R.; MARTINELLI, L. A.. Changes in Soil Carbon, Nitrogen, and Phosphorus Due to Land-Use Changes in Brazil. Biogeosciences, v.12, p.4765-4780, 2015. DOI: http://doi.org/10.5194/bg-12-4765-2015

HEDGES, J. I.; MAYORGA, E.; TSAMAKIS, E.; MCCLAIN, M. E.; AUFDENKAMPE, A.; QUAY, P.; RICHEY, J. E.; BENNER, R.; OPSAHL, S.; BLACK, B.; PIMENTEL, T.; QUINTANILLA, J.; MAURICE, L.. Organic matter in Bolivian tributaries of the Amazon River: A comparison to the lower mainstream. Limnology and Oceanography, v.45, n.7, p.1449-1466, 2000. DOI: http://doi.org/10.4319/lo.2000.45.7.1449

HUANG, T.-H.; FU, Y.-H.; PAN, P.-Y.; CHEN, C.-T. A.. Fluvial Carbon Fluxes in Tropical Rivers. Current Opinion in Environmental Sustainability, v.4, n.2, p.162-169, 2012. DOI: http://doi.org/10.1016/i.cosust.2012.02.004

JIMÉNEZ-MUÑOZ, J. C.; MATTAR, C.; BARICHIVICH, J.; SANTAMARÍA-ARTIGAS, A.; TAKAHASHI, K.; MALHI, Y.; SOBRINO, J. A.; SCHRIER, G.. Record-Breaking Warming and Extreme Drought in the Amazon Rainforest during the Course of El Niño 2015/2016. Scientific Reports, v.6, n.33130, p.1-7, 2016. DOI: http://doi.org/2016. $\underline{10.1038 / \text { srep33130 }}$

LEWIS, W. M.; MELACK, J. M.; MCCLAIN, M.; RICHEY, J. E.. Nitrogen Yields from Undisturbed Watersheds in the Americas. Biogeochemistry, v.46, n.1-3, p.149-162, 1999. 
LIU, J.; BOWMAN, K. W.; SCHIMEL, D. S.; PARAZOO, N. C.; JIANG, Z.; LEE, M.; BLOOM, A. A.; WUNCH, D.;

FRANKENBERG, C.; SUN, Y.; O'DELL, C. W.; GURNEY, K. R.; MENEMENLIS, D.; GIERACH, M.; CRISP, D.; ELDERING, A.. Contrasting Carbon Cycle Responses of the Tropical Continents to the 2015-2016 El Niño. Science, v.358, n.6360, p.eaam5690, 2017. DOI:

http://doi.org/10.1126/science.aam5690

LOVEJOY, T. E.; NOBRE, C.. Amazon Tipping Point. Science Advances, v.4, n.2, p.eaat2340, 2018. DOI: http://doi.org/10.1126/sciadv.aat2340

MANN, P. J.; DAVYDOVA, A.; ZIMOV, N.; SPENCER, R. G. M.; DAVYDOV, S.; BULYGINA, E.; ZIMOV, S.; HOLMES, R. M.. Controls on the Composition and Lability of Dissolved Organic Matter in Siberia's Kolyma River Basin. Journal of Geophysical Research: Biogeosciences, v.117, n.G1, p.G01028, 2012. DOI: http://doi.org/10.1029/2011JG001798

MARENGO, J. .; NOBRE, C. .; TOMASELLA, J.; CARDOSO, M. .; OYAMA, M.. Hydro-climatic and ecological behaviour of the drought of Amazonia in 2005. Philosophical Transactions of the Royal Society B: Biological Sciences, v.363, n.1498, p.1773-1778, 2008. DOI: http://doi.org/10.1098/rstb.2007.0015

MARENGO, J. A.; ALVES, L. M.; SOARES, W. R.; RODRIGUEZ, D. A.; CAMARGO, H.; RIVEROS, M. P.; PABLÓ, A. D.. Two Contrasting Severe Seasonal Extremes in Tropical South America in 2012: Flood in Amazonia and Drought in Northeast Brazil. Journal of Climate, v.26, n.22, p.91379154, 2013. DOI: http://doi.org/10.1175/JCLI-D-12-00642.1

MARENGO, J. A.; ESPINOZA, J. C.. Extreme Seasonal Droughts and Floods in Amazonia: Causes, Trends and Impacts. International Journal of Climatology, v., n.3, p.1033-1050, 2016. DOI: http://doi.org/10.1002/joc.4420

MARENGO, J. A.; TOMASELLA, J.; SOARES, W. R.; ALVES, L. M.; NOBRE, C. A.. Extreme Climatic Events in the Amazon Basin: Climatological and Hydrological Context of Recent Floods. Theoretical and Applied Climatology, v.107, n.1-2, p.73-85, 2012. DOI: http://doi.org/10.1007/s00704-011$\underline{0465-1}$

MARTINELLI, L. A.; COLETTA, L. D.; RAVAGNANI, E. C.; CAMARGO, P. B.; OMETTO, J.; FILOSO, S.; VICTORIA, R. L.. Dissolved nitrogen in rivers: comparing pristine and impacted regions of Brazil. Brazilian Journal of Biology, v.70, n.3, p.709-722, 2010. DOI: http://doi.org/10.1590/S1519$\underline{69842010000400003}$

MARTINELLI, L. A.; DEVOL, A. H.; VICTORIA, R. L.; RICHEY, J. E.. Stable Carbon Isotope Variation in C3 and C4 Plants along the Amazon River. Nature, v.353, n.6339, p.57-59, 1991. DOI: http://doi.org/10.1038/353057a0

MARTINELLI, L. A.; VICTORIA, R. L.; DEVOL, A. H.; RICHEY, J. E.; FORSBERG, B. R.. Suspended Sediment Load in the Amazon Basin: An Overview. GeoJournal, v.19, n.4, p.381389, 1989. DOI: http://doi.org/10.1007/BF00176907

MAYORGA, E.; AUFDENKAMPE, A. K.; MASIELLO, C. A.; KRUSCHE, A. V.; HEDGES, J. I.; QUAY, P. D.; RICHEY, J. E.;
BROWN, T. A.. Young organic matter as a source of carbon dioxide outgassing from Amazonian rivers. Nature, v.436, n.7050, p.538-541, 2005. DOI:

http://doi.org/10.1038/nature03880

MCCLAIN, M. E.; ELSENBEER, H.. Terrestrial inputs to Amazon strems and internal biogeochemical process. In: MCCLAIN, M. E.; VICTORIA, R.; RICHEY, J. E.. The Biogeochemistry of the Amazon Basin. New York: Oxford University Press, 2001.

MCDOWELL, N.; ALLEN, C. D.; ANDERSON-TEIXEIRA, K.; BRANDO, P.; BRIENEN, R.; CHAMBERS, J.; CHRISTOFFERSEN, B.; DAVIES, S.; DOUGHTY, C.; DUQUE, A.; ESPIRITO-SANTO, F.; FISHER, R.; FONTES, C. G.; GALBRAITH, D.; GOODSMAN, D.; GROSSIORD, C.; HARTMANN, H.; HOLM, J.; JOHNSON, D. J.; KASSIM, A. R.; KELLER, M.; KOVEN, C.; KUEPPERS, L.; KUMAGAI, T.; MALHI, Y.; MCMAHON, S. M.; MENCUCCINI, M.; MEIR, P.; MOORCROFT, P.; MULLER-LANDAU, H. C.; PHILLIPS, O. L.; POWELL, T.; SIERRA, C. A.; SPERRY, J.; WARREN, J.; XU, C.; XU, X.. Drivers and Mechanisms of Tree Mortality in Moist Tropical Forests. New Phytologist, v.219, n.3, p.851-869, 2018. DOI:

http://doi.org/10.1111/nph.15027

MILLIMAN, J. D.; MEADE, R. H.. World-Wide Delivery of River Sediment to the Oceans. The Journal of Geology, v.91, n.1, p.1-21, 1983. DOI: http://doi.org/10.1086/628741

MOREIRA-TURCQ, P.; SEYLER, P.; GUYOT, J. L.; ETCHEBER, H.. Exportation of Organic Carbon from the Amazon River and Its Main Tributaries. Hydrological Processes, v.17, n.7, p.1329-1344, 2003. DOI: http://doi.org/10.1002/gbc.20022

NEILL, C.; DEEGAN, L. A.; THOMAS, S. M.; CERRI, C. C.. Deforestation for Pasture Alters Nitrogen and Phosphorus in Small Amazonian Streams. Ecological Applications, v.11, n.6, p.1817-1828, 2001. DOI: https://doi.org/10.1890/10510761(2001)011[1817:DFPANA]2.0.CO;2

NOBRE, C. A.; NOBRE, A. D.. O balanço de carbono da Amazônia brasileira. Estudos Avançados, v.16, n.45, p.81-90, 2002. DOI: http://doi.org/10.1590/S0103$\underline{40142002000200006}$

NOBRE, C. A.; SAMPAIO, G.; BORMA, L. S.; CASTILLA-RUBIO, J. C.; SILVA, J. S.; CARDOSO, M.. Land-Use and Climate Change Risks in the Amazon and the Need of a Novel Sustainable Development Paradigm. Proceedings of the National Academy of Sciences, v.113, n.39, p.10759-10768, 2016. DOI: http://doi.org/10.1073/pnas.1605516113

QUAY, P. D.; WILBUR, D.; RICHEY, J. E.; HEDGES, J. I.; DEVOL, A. H.. Carbon cycling in the Amazon River: Implications from the $13 \mathrm{C}$ compositions of particles and solutes. Limnology and Oceanography, n.4, v.37, p.857-871, 1992. DOI: https://doi.org/10.4319/lo.1992.37.4.0857

RICHEY, J. E.; HEDGES, J. I.; DEVOL, A. H.; QUAY, P. D.; VICTORIA, R.; MARTINELLI, L.; FORSBERG, B. R..

Biogeochemistry of Carbon in the Amazon River. Limnology and Oceanography, v.35, n.2, p.352-371, 1990. DOI: http://doi.org/10.4319/lo.1990.35.2.0352

RICHEY, J. E.; MEADE, R. H.; SALATI, E.; DEVOL, A. H.; NORDIN, C. F.; SANTOS, U. D.. Water Discharge and 
Suspended Sediment Concentrations in the Amazon River: 1982-1984. Water Resources Research, v.22, n.5, p.756-

764, 1986. DOI: http://doi.org/10.1029/WR022i005p00756

RICHEY, J. E.; MELACK, J. M.; AUFDENKAMPE, A. K.; BALLESTER, V. M.; HESS, L. L.. Outgassing from Amazonian rivers and wetlands as a large tropical source of atmospheric CO2. Nature, v.416, n.6881, p.617-620, 2002. DOI: http://doi.org/10.1029/WR022i005p00756

RICHEY, J. E.; VICTORIA, R. L.; SALATI, E.; FORSBERG, B. R.. The Biogeochemistry of a Major River System: The Amazon Case Study. Chichester: John Wiley \& Sons, 1991.

SATYAMURTY, P.; DA COSTA, C. P. W.; MANZI, A. O.. Moisture Source for the Amazon Basin: A Study of Contrasting Years. Theoretical and Applied Climatology, v.111, n.1-2, p.195-209, 2013. DOI: http://doi.org/10.1007/S00704-012-0637-7

SATYAMURTY, P.; DA COSTA, C. P. W.; MANZI, A. O.; CANDIDO, L. A.. A Quick Look at the 2012 Record Flood in the Amazon Basin. Geophysical Research Letters, v.40, n.7, p.1396-1401, 2013. DOI: http://doi.org/10.1007/s00704012-0637-7

SIOLI, H.. The Amazon. Dordrecht: Springer Netherlands, 1984.

SOLAR, R. R. C.; BARLOW, J.; ANDERSEN, A. N.; SCHOEREDER, J. H.; BERENGUER, E.; FERREIRA, J. N.; GARDNER, T. A.. Biodiversity consequences of land-use change and forest disturbance in the Amazon: A multi-scale assessment using ant communities. Biological Conservation, v.197, p.98-107, 2016. DOI: http://doi.org/10.1016/j.biocon.2016.03.005

WARD, N. D.; KRUSCHE, A. V.; SAWAKUCHI, H. O.; BRITO, D. C.; CUNHA, A. C.; MOURA, J. M. S.; SILVA, R.; YAGER, P. L.; KEIL, R. G.; RICHEY, J. E.. The Compositional Evolution of Dissolved and Particulate Organic Matter along the Lower Amazon River: Óbidos to the Ocean. Marine Chemistry, v.177, p.244-256, 2015. DOI:

http://doi.org/10.1016/j.marchem.2015.06.013

A CBPC - Companhia Brasileira de Produção Científica (CNPJ: 11.221.422/0001-03) detém os direitos materiais desta publicação. Os direitos referem-se à publicação do trabalho em qualquer parte do mundo, incluindo os direitos às renovações, expansões e disseminações da contribuição, bem como outros direitos subsidiários. Todos os trabalhos publicados eletronicamente poderão posteriormente ser publicados em coletâneas impressas sob coordenação da Sustenere Publishing, da Companhia Brasileira de Produção Científica e seus parceiros autorizados. Os (as) autores (as) preservam os direitos autorais, mas não têm permissão para a publicação da contribuição em outro meio, impresso ou digital, em português ou em tradução. 